Case report

\title{
Primary Female Urethral Cancer: A Case Report and Review of Literature
}

\author{
Yupeng Guan 1, Gang Yu 1, Qianyun Tang',2, Huan Zhou' ${ }^{3}$, Zhiming Bai 1,* \\ 1 Department of Urology, Affiliated Haikou Hospital, Xiangya School of Medicine, Central South University, \\ Haikou 570208, China; kwanyp90@163.com; ydna2@yahoo.com; 1888958017@163.com \\ 2 Xiangya Nursing School, Central South University, Changsha, 410006, China; 1888958017@163.com \\ 3 Department of Pathology, Affiliated Haikou Hospital, Xiangya School of Medicine, Central South \\ University, Haikou 570208, China; 550745652@qq.com \\ * Correspondence: hkbzm2018@aliyun.com; Tel.: +86-0898-66151224
}

\begin{abstract}
Primary urethral malignancies are rare in clinical practice and elderly female are prone to suffer. The cause of PUC is unknown and may be related to long-term chronic inflammation, infection, sexual intercourse, pregnancy and irritation of the urethra. Incipient symptom of PUC is not distinctness and can easily lead to misdiagnosis. The final diagnosis relied on transvaginal ultrasound-guided mass biopsy and urethral biopsy. Early tumor treatment is mainly based on surgical resection. For advanced tumors, it is recommended to adopt a comprehensive treatment plan combining surgery, radiotherapy and chemotherapy We report this case with the aim of bringing attention to that primary urethral cancer progresses rapidly and has a poor prognosis, it is urged to call for a need of diagnostic and treatment specification.
\end{abstract}

Keywords: Primary urethral cancer; Elderly female; Prognosis

\section{Introduction}

Primary urethral cancer (PUC) is a malignant tumor that is covered by the urethra and covered with epithelium and glands. It is rare in clinical practice and accounts for less than $1 \%$ of urinary tract malignancies[1]. Since Boaviin and Deuges first reported primary female urethral cancer in 1833 , about 1,300 cases have been reported worldwide. Both sexes can occur, and the ratio of male to female is about 1:50, which is common in the elderly. Urethral cancer may have a variety of histological types, including squamous cell carcinoma, transitional cell carcinoma, adenocarcinoma, undifferentiated carcinoma, sarcoma, and melanoma[2].

The cause of PUC is unknown and may be related to long-term chronic inflammation, infection, sexual intercourse, pregnancy and irritation of the urethra. Incipient symptom of PUC is not distinctness and can easily lead to misdiagnosis. Common symptoms are urethral blood secretions, bleeding, urinary tract irritation. In the advanced stage, the mass is cauliflower-like and foul-smelling. Symptoms may include pelvic pain, abscess around the urethra, urinary incontinence, urethral fistula, and urinary retention. Minority of patients were asymptomatic and tumors were found by chance due to examination for other diseases or annual body check. Special attention should be paid to inguinal lymph nodes and double-diagnosis (female) during physical examination. $20 \%-57 \%$ of newly diagnosed patients have inguinal lymphadenopathy, $90 \%$ of which are cancer metastasis. Computed tomographic (CT) and Magnetic Resonance Imaging (MRI) help to understand the extent of tumor infiltration and whether there is pelvic lymph node metastasis. Any proximal urethral cancer needs to be alert to bladder cancer[3].

\section{Case presentation}

A 65-year-old female was presented to our hospital with a 2-month history of difficulty in urinating and was found right kidney hydronephrosis for 2 weeks. Two months ago, the patient did not have obvious incentives to start with difficulty in urinating, frequency, urgency, dysuria 
and urinary tract became thinning, no gross hematuria, the above symptoms did not significantly relieve after anti-infective treatment in local clinic. CT scan for urinary system in the local clinic prompted severe hydronephrosis in the right kidney, dilatation in the middle and upper segments and obstruction in the lower segment of right ureter (no film is provided); Lumbar MRI suggests: L5/S1 left nerve root localized thickening and bulging, disc herniation in L5/S1 and L3/4/5. The patient was transferred to our hospital for further diagnosis and treatment. Her general physical examinations were negative except for poor nutritional status. No abnormality in the appearance of the vulva. The indwelling catheter was circulated smoothly, no obvious hematuria was observed, and there was no abnormal secretion in the external urethra. Vaginal touch examination can reach a hard mass in the anterior wall of the vagina, located in the posterior urethral bladder neck, fixed and no tenderness. Past medical history of tubal ligation and no history of vaginal bleeding. She has been menopause and no special genetic history in the family.

Urine routine suggests white blood cells $45 / \mathrm{HP}$, occult blood negative, and urinary bacteria cultured as Enterococcus faecalis, which is a multi-drug resistant strain. The urine culture was negative, and the urine white blood cells were 7/HP after 6-day antibiotic treatment according to the urine culture and drug susceptibility results. Urodynamic examination revealed urethral or bladder outlet obstruction and normal detrusor contractility. CT (Figure 1) showed a $2.7^{*} 2.2 \mathrm{~cm}$ mass of lumps around the urethra and wrapped around the urethra, significantly strengthened after enhancement. Colposcopy showed no obvious organic lesions in the cervix, and no malignant lesions were seen in the cervical biopsy. Urethral cystoscopy and right ureteroscopy showed that the urethral mucosa was pale and erosive, no obvious new organisms were found, and no new organisms were found in the bladder. The left ureteral orifice is not clear, and the right ureter is stenosis. no new organisms in the ureter below the stenosis, ureteroscopy difficult to pass, the replacement of the smaller diameter of the ureteroscopy still cannot pass, but the F5 ureteral catheter can pass the stenosis to the renal pelvis, the final stenosis failed to take pathological biopsy. Indwelling the right ureteral catheter to the renal pelvis drainage. Retracting ureteroscopy, and the biopsy forceps were used to take a pathological examination of the urethral mucosa about $3 * 3 \mathrm{~mm}$. Transvaginal ultrasonography was performed to observe a large mass around the urethra, about $3^{*} 3^{*} 2 \mathrm{~cm}$, and a needle biopsy was performed at $2,4,8$, and 10 o'clock in the urethra under ultrasound guidance. Retrograde angiography showed obvious stenosis in the middle and lower segment, and the stenosis segment was about $3.7 \mathrm{~cm}$ long with no filling defect after indwelling the right ureteral catheter. Postoperative pathological findings suggest: a small amount of puncture tissue (Figure 2 and Figure 3), see the heterogeneous cells arranged in a beam-like and agglomerate invasive growth. Immunohistochemistry: CK7 (+), CK5/6 (+/-), CK20 (+), CK8/18 (+), P63 (+), CEA (-), Vimentin (-). Pathological diagnosis: (in addition to the urethra) invasive urothelial carcinoma, high level.

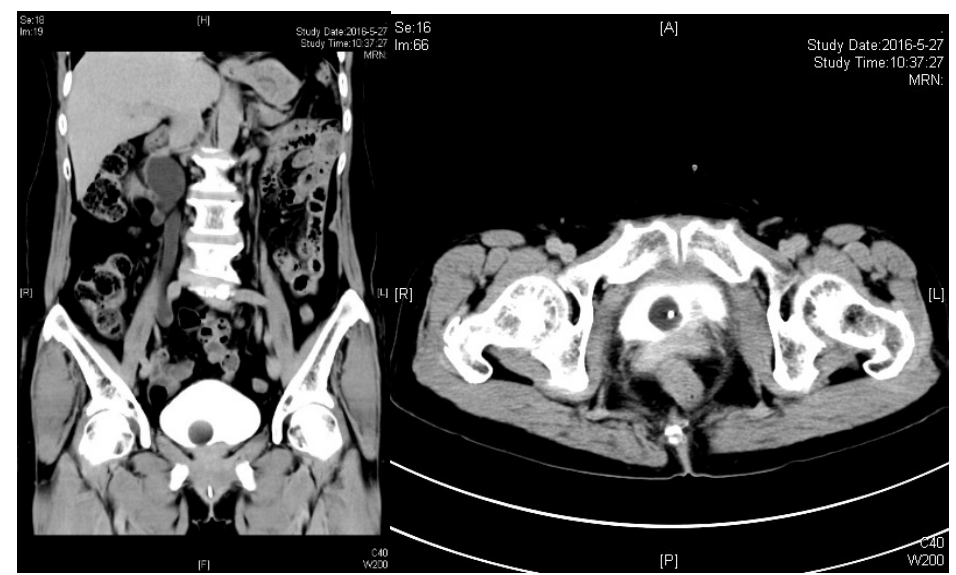

Figure 1. Near the urethra, a $2.7^{*} 2.2 \mathrm{~cm}$ mass shadow was seen and wrapped around the urethra. CT enhanced significantly. 


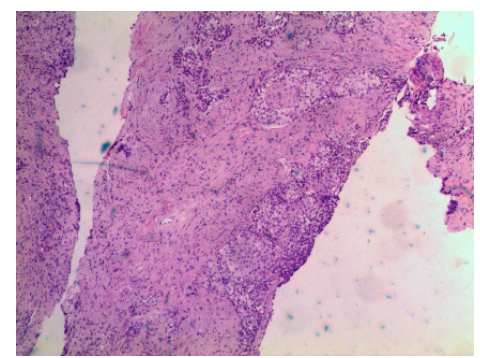

Figure 2 . The heterotypic cells are arranged in a beam-like and agglomerate invasive growth. HE staining $\times 400$

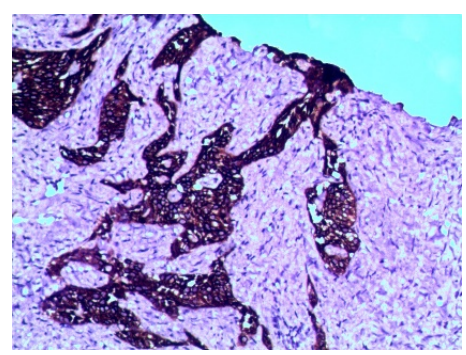

Figure 3. Cover squamous epithelium, partial necrosis, partial mild atypical hyperplasia, interstitial cell infiltration. CK7 10×10

We offer patients two treatment options: one, radical surgery: the scope of resection includes the bladder, urethra, anterior wall of the vagina, uterus and ovaries, while clearing the pelvic lymph nodes and diverting the urine. The disadvantage is that bleeding is easy during and after surgery, and the quality of life may be significantly reduced after surgery. Another, radiotherapy and chemotherapy, external irradiation combined with doxorubicin, cisplatin and other chemotherapy drugs, but cost expensively. After communicating with patients and their families, they refused surgery or radiotherapy and chemotherapy and were discharged. Follow-up to 2 months after discharge, the patient died considering of tumor progression.

\section{Discussion}

The characteristics of this case, the patient is an elderly woman, admitted to hospital due to dysuria, the initial symptoms are not obvious, no hematuria, no new organisms in the external urethra, only the urethra palpation is stiff. No new organisms were seen in the urethra and bladder under the urethra cystoscopy. CT shows that a prostate-like mass around the urethra surrounds the urethra and needs to be differentiated from Skene's adenocarcinoma[4]. Local clinic CT showed patients with lumbar disc herniation, and we used urodynamic examination to exclude urinary retention caused by neurogenic bladder. Colposcopy was performed to exclude gynecologic tumors. Retrograde ureteroscopy angiography confirms the cause of hydronephrosis in the right kidney. The final diagnosis relied on transvaginal ultrasound-guided mass biopsy and urethral biopsy.

It is open to discuss whether the diagnosis of carcinoma of the urethra is primary or metastatic. If it is metastatic urethral cancer, it mainly comes from the urothelium, which is the renal pelvis, ureter and bladder from top to bottom. The clinical symptoms of upper urinary tract tumors generally include hematuria, lumbar pain, and abdominal mass. 30\% to 50\% of upper urinary tract tumors can be combined with bladder tumors. Retrograde urography may show a defect in the renal pelvis or ureter filling, which is characteristics of upper urinary tract tumors. None of the above features were found in this case. Therefore, although the biopsy was not taken at the ureteral stricture, the tumor may not be absolutely excluded, that is, the possibility of urethral cancer due to metastasis of upper urinary tract tumors cannot be completely ruled out. However, the right ureteral stenosis, no obvious space occupying imaging, retrograde urography without filling defect, combined with the patient's history of previous tubal ligation, the authors considered that the right 
ureteral stricture is more likely to be associated with the history of previous surgery. In summary, this case is considered as primary urethral cancer.

The histological type of urethral cancer, progression of the disease, choice of treatment and prognosis depend to a large extent on the urethral segment of the lesion. Distal PUC, that is, the first third of the urethra, the tumor stage is lower[5], and the PUC occurring in the proximal urethra are mostly whole urethral invasion with definitive pelvic lymph node metastasis, the stage is higher, and the prognosis is also poor[6], which may be related to the anatomical features of the urethra and the lymphatic drainage pathway. Early tumor treatment is mainly based on surgical resection. Distal PUC has a good treatment effect and $90 \%$ patients are expected to survive. The prognosis of distal PUC is poor[7]. Generally, radical cystectomy is performed. The scope of resection includes bladder, vagina, anterior wall of the vagina, uterus and ovaries. At the same time, pelvic lymph nodes are removed and urinary diversion is performed. Prophylactic lymphadenectomy is controversial. If pubic invasion, pubic symphysis can be removed. The 5-year survival rate is $10 \%-17 \%$, and most patients have local recurrence, which is due to infection, bleeding and cachexia. For advanced tumors, it is recommended to adopt a comprehensive treatment plan combining surgery, radiotherapy and chemotherapy[8].

Female primary urethral cancer is difficult to distinguish with benign tumors such as urethral polyps and urethral meat mites. The former is not obvious pain, the texture is hard, the color is pale or gray, and if the tumor is necrotic or infection, it will be accompanied by stench, and the vagina or vestibule can touch the stiff tumor. The diagnosis should be combined with clinical symptoms, digital examination, auxiliary examination and biopsy[9]. Since a small quantity of accumulated cases in this disease, its treatment methods and levels are significantly behind other tumors, and there is currently no uniform standardized treatment plan.

\section{Conclusions}

Primary urethral cancer is rare and elderly female are prone to suffer. The EAU guidelines suggest that advanced age (age $>65$ years), high stage, lymph node metastasis, distant metastasis, and large tumor volume are all factors suggesting poor prognosis[10]. We report this case with the aim of bringing attention to that primary urethral cancer progresses rapidly and has a poor prognosis, it is urged to call for a need of diagnostic and treatment specification.

Author Contributions: conceptualization, Yupeng Guan and Gang Yu; validation, Yupeng Guan, Gang Yu, Qianyun Tang, Huan Zhou and Zhiming Bai; Immunophenotypic Analysis, Huan Zhou; writing-original draft preparation, Yupeng Guan; writing - review and editing, Zhiming Bai; supervision, Zhiming Bai; All authors read and approved the final manuscript.

Funding: This research received no external funding

Acknowledgments: No grant support needs to be reported.

Conflicts of Interest: The authors declare no conflict of interest.

\section{References}

1. Dayyani, F.; Hoffman, K.; Eifel, P.; Guo, C.; Vikram, R.; Pagliaro, L.C.; Pettaway, C. Management of advanced primary urethral carcinomas. BJU international 2014, 114, 25-31, doi:10.1111/bju.12630.

2. Zhang, M.; Adeniran, A.J.; Vikram, R.; Tamboli, P.; Pettaway, C.; Bondaruk, J.; Liu, J.; Baggerly, K.; Czerniak, B. Carcinoma of the urethra. Hum Pathol 2018, 72, 35-44, doi:10.1016/j.humpath.2017.08.006.

3. Gakis, G.; Efstathiou, J.A.; Daneshmand, S.; Keegan, K.A.; Clayman, R.H.; Hrbacek, J.; Ali-El-Dein, B.; Zaid, H.B.; Schubert, T.; Mischinger, J., et al. 
Oncological Outcomes of Patients with Concomitant Bladder and Urethral Carcinoma. Urol Int 2016, 97, 134-141, doi:10.1159/000448335.

4. Thum, S.; Haben, B.; Christ, G.; Sen Gupta, R. [Female prostate cancer?]. Pathologe 2017, 38, 448-450, doi:10.1007/s00292-017-0322-9.

5. Traboulsi, S.L.; Witjes, J.A.; Kassouf, W. Contemporary Management of Primary Distal Urethral Cancer. Urol Clin North Am 2016, 43, 493-503, doi:10.1016/j.ucl.2016.06.010.

6. Zinman, L.N.; Vanni, A.J. Management of Proximal Primary Urethral Cancer: Should Multidisciplinary Therapy Be the Gold Standard? Urol Clin North Am 2016, 43, 505-513, doi:10.1016/j.ucl.2016.06.011.

7. Gakis, G.; Morgan, T.M.; Efstathiou, J.A.; Keegan, K.A.; Mischinger, J.; Todenhoefer, T.; Schubert, T.; Zaid, H.B.; Hrbacek, J.; Ali-El-Dein, B., et al. Prognostic factors and outcomes in primary urethral cancer: results from the international collaboration on primary urethral carcinoma. World J Urol 2016, 34, 97-103, doi:10.1007/s00345-015-1583-7.

8. Gakis, G.; Morgan, T.M.; Daneshmand, S.; Keegan, K.A.; Todenhofer, T.; Mischinger, J.; Schubert, T.; Zaid, H.B.; Hrbacek, J.; Ali-El-Dein, B., et al. Impact of perioperative chemotherapy on survival in patients with advanced primary urethral cancer: results of the international collaboration on primary urethral carcinoma. Ann Oncol 2015, 26, 1754-1759, doi:10.1093/annonc/mdv230.

9. Kang, M.; Jeong, C.W.; Kwak, C.; Kim, H.H.; Ku, J.H. Survival Outcomes and Predictive Factors for Female Urethral Cancer: Long-term Experience with Korean Patients. J Korean Med Sci 2015, 30, 1143-1149, doi:10.3346/jkms.2015.30.8.1143.

10. Gakis, G.; Witjes, J.A.; Comperat, E.; Cowan, N.C.; De Santis, M.; Lebret, T.; Ribal, M.J.; Sherif, A.M.; European Association of, U. EAU guidelines on primary urethral carcinoma. Eur Urol 2013, 64, 823-830, doi:10.1016/j.eururo.2013.03.044. 